\title{
A systems biology view of the development of new antimicrobial therapies
}

\section{Introduction}

Antimicrobial resistance has received an increased level of attention in the recent years due its alarming intensification and spread worldwide. Despite acknowledgment of the acceleration of antimicrobial resistance, efforts dedicated to new antibiotics discovery and development or optimization of current therapies have declined in the past two decades. Following the report on antibiotic resistance threats in the US in 2013 from the $\mathrm{CDC}^{1}$ and the distressing report of the WHO on the post-antibiotic era ${ }^{2}$ in 2014, controlling the development and spread of antibiotic resistance became a top US security and public health priority for the Obama Administration with the release of its National Strategy on Combating Antibiotic-Resistant Bacteria in September 2014. ${ }^{3}$

The development of antimicrobial resistance is inevitable and inherent to the remarkable mechanisms of bacterial adaptation to adverse environmental conditions in order to survive. However, many human actions have contributed to the currently observed acceleration and spread of antimicrobial resistance (poor hygiene, over prescription of antibiotics, intensive use of antibiotics in farming, etc.) and battling the antimicrobial resistance threat will require scientific, societal, and political actions to be taken. In addition to changes in human and veterinarian health care practices and new policy development for better risk assessment, there is still a crucial need for the development of new antimicrobial therapies. The number of FDA-approved antibiotics has been constantly decreasing since the 1990s and biopharmaceuticals companies are less willing to invest in the drug pipeline, ${ }^{4}$ especially for a product that bacterial resistance will eventually render obsolete. According to a Pew Charitable Trusts study, only 38 new antibiotics were in development by pharmaceutical companies in 2014. ${ }^{5}$ Despite the gap in the drug development pipeline, there are very promising approaches currently being applied that have led to encouraging findings in the recent years.

Antibiotics are classified based on their drug-target interactions leading to the inhibition of cell growth (bacteriostatics) or induction of bacterial cell death (bacteriocides). The modes of action have been very well studied and fall into three major classes: inhibition of DNA replication or repair, inhibition of protein synthesis, and inhibition of cell-wall biosynthesis and turnover. ${ }^{6}$ It has however become very clear that the antibiotics' mechanisms of action are highly complex and their mode of action goes beyond the primary interaction target. An understanding of the bacterial global response that occurs as a result of the primary antibiotic action is still elusive. Technical advancements in the fields of genomics, transcriptomics, proteomics and metabolomics have provided tools to assess responses to a multitude of external and internal perturbations. The dissection of these mechanisms requires equally complex experimental design and data collection and analysis, which can only be achieved with increased efforts in computational biology, bioinformatics, and overall systems biology approaches. The effect of antibiotics can now be monitored at the various cellular levels (genome, transcriptome, proteome, metabolome) and in a dynamic way (fluxome). Very exciting research on the mechanism of bacterial cell death induced by antimicrobial action is currently using
Volume I Issue I - 2015

\author{
Heidi Vitrac \\ Department of Biochemistry and Molecular Biology, University \\ of Texas Medical School-Houston, USA
}

\begin{abstract}
Correspondence: Heidi Vitrac, Department of Biochemistry and Molecular Biology, University of Texas Medical SchoolHouston, 643I Fannin St, Houston, TX 77030, USA, Tel 7|35006I20, Fax 7|35000652,Email heidi.vitrac@uth.tmc.edu
\end{abstract}

Received: April 23, 2015 | Published: April 27, 2015

systems biology approaches combining metabolomics-based strategy, network modeling, and analysis with biochemical characterizations. Pioneering work from groups like James Collins' at MIT led to a more detailed understanding of these mechanisms. ${ }^{7}$

Their work presents an integrated view of the consequences of antibiotics treatment on the cell: impacts on the central metabolism (TCA cycle, oxidative phosphorylation, etc.), the membrane potential, protein synthesis and quality control, production of reactive oxygen species (ROS), and stress responses have been uncovered. It appears that various classes of bactericidal compounds - acting on different classes of targets - induce cell death through a shared mechanism involving the formation of hydroxyl radicals. Antibiotic treatment triggers a chain of events in the cell that leads to an increase in NADH oxidation with stimulation of the TCA cycle, overproduction of superoxide anion at the electron transport chain level that can damage Fe-S clusters, ultimately releasing ferrous iron and increasing the production of hydroxyl radicals through the Fenton reaction. ${ }^{7}$ It has been well established that the bacterial physiological homeostasis is a fine-tuned process with tightly controlled and regulated components that are highly interconnected. With the expansion of the use of multiomics techniques, bacterial physiology ${ }^{8}$ and adaptation to stress has been investigated in more detail using integrated approaches. ${ }^{9-11}$ However, only a few studies integrate a systemic approach to elucidate the global effect of antibiotics on bacteria. It can be anticipated that such studies will flourish in the coming years.

The use of synthetic biology and in silico modeling adds a predictive aspect that allows identification of potential targets to be modulated. The establishment of the participation of ROS during antibiotic-induced bacterial cell death paved the way towards improvement strategies of established therapies. Network modeling and predictions of ROS formation upon genetic or chemical perturbations of the cell led to the identification of potential protein targets (enzymes involved in the central metabolism such as succinate dehydrogenase)..$^{12}$ Experimental validation of the synergistic effects of antibiotic treatment with chemical inhibition of the target is a promising lead towards the development of adjuvant therapies. The 
predictive value of metabolic models should be more broadly used to facilitate the establishment of chemical library screening aiming at discovering compounds that enhance the killing efficacy of current antibacterial agents. This approach already led to the uncovering of potential therapeutic strategies that does not require new antibiotics discovery. ${ }^{12,13}$

Anew line of reports inspired by this type of approach is increasingly emerging where antimicrobial action is monitored and perturbations in the systems are attempted to enhance the efficacy of already established therapies. Strong evidence suggests that susceptibility to antimicrobial compounds is highly correlated to the metabolic state of the cell, and particular metabolic profiles are associated with certain antibiotic resistances. It has been suggested that alteration or reversal of this resistant-prone metabolic state could improve antibiotic efficiency. ${ }^{14}$ This has proven to be particularly successful in the case of bacterial persisters. Reactivation of bacterial metabolism (switch from dormant to metabolically active bacteria) by a single metabolite restores the efficacy of the antibiotics. ${ }^{12,13,15}$ Similarly, discovery of the involvement of oxidative stress in the mechanism of action of bactericides has led to the use of ROS stimulating molecules as codrugs to enhance the killing potential of antibiotics. ${ }^{12,13}$ Advances in systems biology that combine modelling with experimentation and advances in techniques that allow dynamic physiological processes to be followed have shifted the field of bacteriology. The combined use of antibiotics and metabolic reprogramming through metabolites is a promising approach in the development of improved therapies.

On a different front, the recent discovery of a new antibiotic compound (teixobactin) also brings some hope in the fight against bacterial resistance. ${ }^{16}$ In addition to being the first new class of antibiotics discovered in the past 30 years, this compound acts by binding to the glycolipid component of the cell wall. Other antibiotics mediate their effects through interactions with proteins; bacteria might be less efficient at developing resistance to this mode of action. More interestingly, this new compound has been discovered in cellular communities that are almost impossible to culture under traditional microbiological conditions in the laboratory. Our knowledge of the bacterial world is indeed limited by our ability to study the microorganisms in laboratory-compatible conditions. Many specimens stemming from the soil do not thrive in petri dishes and first need to be "domesticated" therefore limiting their use by scientists. It is estimated that only $1 \%$ of soil bacteria have been cultured in laboratory conditions. The development of microfluidics devices such as the iChip ${ }^{16}$ is a very exciting technological advancement allowing researchers to culture bacteria in their "native habitat". It clearly opens new avenues in microbiological studies and offers promise in the discovery of new molecules.

Fighting antibacterial resistance may never reach a true endpoint and will remain a permanent struggle since bacteria can evolve and adapt very quickly to environmental stresses. The systems biology approach has however proven extremely efficient at understanding and deciphering the complexity of the physiological alterations occurring in bacteria during exposure to antibiotics. Discovery of new antibiotics combined with network modulation of the metabolic status of the cell could constitute a new and sustainable battle plan. These progresses should encourage the scientific community to integrate this global vision in their research.

\section{Acknowledgements}

None.

\section{Conflict of interest}

The author declares no conflict of interest.

\section{References}

1. Centers for Disease Control and Prevention (CDC). Antibiotic Resistance Threats in the United States, 2013. USA: Antibiotic/Antimicrobial resistance threat report; 2013.

2. World Health Organization (WHO). Antimicrobial resistance: global report on surveillance 2014. Geneva: World Health Organization technical report series; 2014.

3. The White House. National strategy for combating antibiotic-resistant bacteria. Washington USA; 2014.

4. Cooper MA, Shlaes D. Fix the antibiotics pipeline. Nature. 2011;472(7341):32.

5. The Pew Charitable Trusts. Tracking the Pipeline of Antibiotics in Development. USA: Antibiotic Resistance Project; 2014.

6. Dwyer DJ, Collins JJ, Walker GC. Unraveling the physiological complexities of antibiotic lethality. Anпи Rev Pharmacol Toxicol. $2015 ; 55: 313-332$.

7. Kohanski MA, Dwyer DJ, Hayete B, et al. A common mechanism of cellular death induced by bactericidal antibiotics. Cell. 2007;130(5):797810 .

8. Yoon SH, Han MJ, Jeong H, et al. Comparative multi-omics systems analysis of Escherichia coli strains B and K-12. Genome Biol. 2012;13(5):R37.

9. Jozefczuk S, Klie S, Catchpole G, et al. Metabolomic and transcriptomic stress response of Escherichia coli. Mol Syst Biol. 2010;6:364.

10. Topfer N, Jozefczuk S, Nikoloski Z. Integration of time-resolved transcriptomics data with flux-based methods reveals stress-induced metabolic adaptation in Escherichia coli. BMC Syst Biol. 2012;6:148.

11. Krin E, Danchin A, Soutourina O. Decrypting the H-NS-dependent regulatory cascade of acid stress resistance in Escherichia coli. BMC Microbiol. 2010;10:273

12. Brynildsen MP, Winkler JA, Spina CS, et al. Potentiating antibacterial activity by predictably enhancing endogenous microbial ROS production. Nat Biotechnol. 2013;31(2):160-165.

13. Grant SS, Kaufmann BB, Chand NS, et al. Eradication of bacterial persisters with antibiotic-generated hydroxyl radicals. Proc Natl Acad Sci US A. 2012;109(30):12147-12152.

14. Kohanski MA, Dwyer DJ, Collins JJ. How antibiotics kill bacteria: from targets to networks. Nat Rev Microbiol. 2010;8(6):423-435.

15. Peng B, Su YB, Li H, et al. Exogenous alanine and/or glucose plus kanamycin kills antibiotic-resistant bacteria. Cell Metab. 2015;21(2):249261.

16. Ling LL, Schneider T, Peoples AJ, et al. A new antibiotic kills pathogens without detectable resistance. Nature. 2015;517(7535):455-459. 